\title{
Reprocessing of Catheters Used for Coronary Angiography and Changes in the Polymeric Structures
}

\author{
Flavia Myrna ${ }^{a}$, Dinaldo C. Oliveira ${ }^{a}$, b, c, Gloria Vinhas ${ }^{a}$, Ivo Silva ${ }^{a}$, \\ Magno Teixeira ${ }^{\mathrm{a}}$, Andre Galembeck ${ }^{\mathrm{a}}$
}

\begin{abstract}
Background: Catheters are extensively used for coronary angiographies. These devices are long, thin, and flexible tubes of variable diameters that are made of the following polymer compounds: polyamide, polyurethane and Teflon. The objectives of this study are to identify changes that occur in the polymeric structure of the catheters, including an analysis of microcracks, fissures, cuts, perforations, roughness, and porosity in the internal and external layers after the first, second, third, and seventh reprocessing.
\end{abstract}

Methods: Five catheters of the same brand and manufacturer were selected for each analysis, except for the new and seventh catheters, which had only one sample. The catheters that we analyzed included new catheters, catheter reprocessed once $(1 \mathrm{st})$, catheters reprocessed twice (2nd), catheters reprocessed three times (3rd), and catheters reprocessed up to seven times ( 7 th). Samples were cut into small pieces and the scanning electron microscope and infrared spectroscopy were used for analysis.

Results: Scanning electron microscopy (SEM) of reprocessed catheters revealed rougher surface with porosity, stainless steel wires, peeling of the inner layers, micropores, small holes, disordered cracks, cracks, grooves, microcracks, granular appearance, dots whitish, diffuse microcracks, desquamation, diffuse recesses, depressions, protrusions and deep crevices. Infrared spectroscopy suggested a possible change in the chemical structures of the polymer. There was a directly proportional relationship between the reprocessing number and the carbonyl ratio.

Conclusions: The combined SEM and infrared analyses reveal that reprocessing damages the integrity of coronary angiography catheters. Therefore, the reprocessing of such catheters is not recommended.

Keywords: Coronary angiography; Coronary artery disease; Cath-

Manuscript submitted August 31, 2018, accepted September 11, 2018

aFederal University of Pernambuco, Av. Prof. Moraes Rego, 1235, University City, Postal Code 50670901, Recife, PE, Brazil

${ }^{\mathrm{b}}$ Clinical Hospital, Federal University of Pernambuco, Av. Prof. Moraes Rego, 1235, University City, Postal Code 50670901, Recife, PE, Brazil

${ }^{\mathrm{c} C}$ Corresponding Author: Dinaldo C Oliveira, Street Irene Ramos Matos, 68, Postal Code 51011539, Recife, PE, Brazil. Email: dinaldo@cardiol.br

doi: https://doi.org/10.14740/cr775w eters; Scanning electron microscopy; Infrared spectroscopy

\section{Introduction}

Modern cardiology has revolutionized disease management through the introduction of more accurate diagnostic strategies and more effective and less invasive treatment techniques. The procedures that are performed in the hemodynamic laboratories of cardiology referral centers are relevant for the diagnosis and treatment of cardiovascular diseases; however, the associated technology and equipment are costly [1].

One of the most common hemodynamic procedures is coronary angiography (cine-coronary angiography). The statistical data from a developed country show that $1,500,000$ coronary angiographies and 800,000 percutaneous coronary interventions are performed annually. In modern cardiology, pre-shaped catheters are extensively used for coronary angiographies. These devices are long, thin, and flexible tubes of variable diameters that are made of the following polymer compounds: polyamide, polyurethane, and Teflon. The most common devices are $2 \mathrm{~mm}$ wide and $1 \mathrm{~m}$ long and are inserted through the femoral or radial arteries [2].

In general, according to manufacturers' guidelines around the world, catheters for coronary angiography should not be reprocessed and are therefore considered single-use devices. Changes in hemodynamic catheter physical and chemical compositions may occur when they are reprocessed. However, single-use device reprocessing is used for dental, medical, and hospital devices, including hemodynamic and electrophysiology catheters. The reprocessing follows the following steps: 1) visual inspection; 2) washing; 3 ) cleansing, since the effective removal of organic matter with consequent reduction of microbial load and elimination of pyrogens and biofilm is directly related to maintaining the molecular and microstructural integrity of the polymers that are used in single-use materials; 4) disinfection and preparation; 5) packaging; 6) labeling; 7) sterilization; 8) biological and chemical testing; 9) residual analysis of the sterilizing agent, which aims to maintain mechanical functionality, integrity, and quality of these devices after reprocessing [3, 4].

Unfortunately, limited healthcare resources are available in many countries, which motivate the search for inexpensive health strategies and hospital devices. The reuse of coronary 
angiography catheters has been described in the past; therefore, we believe that a scientific evaluation of the changes that occur in pre-shaped catheters that are used in coronary angiography is necessary.

The objectives of this study are to identify changes that occur in the polymeric structure of the catheters, including an analysis of microcracks, fissures, cuts, perforations, roughness, and porosity in the internal and external layers after the first, second, third, and seventh reprocessing. We also analyzed the polymer structure of catheters and its mechanical functionality.

\section{Materials and Methods}

This study was carried out at the Laboratory of Polymeric Materials and Characterization (LMPC) of centro de Tecnologias Estrategicas do Nordeste (CETENE) and Fundacao Oswaldo Cruz (FIOCRUZ). In this study, angiographic catheters that are used during coronary angiography were evaluated.

Five catheters of the same brand and manufacturer were selected for each analysis, except for the new and seventh catheters, which had only one sample. The catheters that we analyzed included new catheters, catheter reprocessed once (1st), catheters reprocessed twice ( $2 \mathrm{nd})$, catheters reprocessed three times (3rd), and catheters reprocessed up to seven times (7th). Judkins left (JL) and Judkins right (JR) catheters that were preshaped in the distal end region were used to access the left coronary artery ostium during the cardiovascular angiographic studies.

Many analyses were carried out to characterize the catheters' chemical, physical, and morphological characteristics, functionality, integrity, and modifications after reprocessing.

\section{Reprocessing}

All catheters were sterilized in the factory using ethylene oxide and packed in clear plastic Tyvek ${ }^{\circledR}$ (DuPont trademark) packaging. The reprocessing phases were performed as follows. In the first phase, the catheter was cleaned using a heparinized solution to remove blood residues. In the second phase, the lumen was irrigated, and in the third phase, the external part of the catheter was cleaned manually. In the fourth phase, the catheters were immersed in an antiseptic solution. In the fifth phase, the catheters were removed from the antiseptic solution and dried, first at ambient temperature and then in compressed air at variable pressure, as required. Catheters were subsequently sterilized using hydrogen peroxide plasma gas.

\section{Scanning electron microscopy (SEM)}

The scanning electron microscope that was used in this analysis was performed using the following criteria: JSM $5600^{\circledR}$ Jeol/EO version 1.0, Accel volt 15, MG 4000, MAG 4000, SEI signal, micro-marker $5 \mu \mathrm{m}$, spot size 33, film number 0000 , wd 10, vacuum, general operator, model Quanta 200 FEG.
Five catheter samples were evaluated, with one new catheter sample and four samples from catheters that were reprocessed one, two, three, or seven times. Samples were cut into small pieces of about $3 \mathrm{~cm}$, which were mounted on stubs (plates where samples were fixed to be read under the SEM) using double carbon tape and coated with gold using a sputter coater. The images were obtained from cuts in the catheters, and this technique was used to protect the existing artifacts from the reprocessing procedure. The same cut location and direction was used for all catheters, to standardize the technique and provide a starting point, since no other techniques were performed in the analysis area.

\section{Infrared spectroscopy}

A Vertex 70 - BRUKER ${ }^{\circledR}$ spectrometer was used to analyze the Fourier-transform infrared (FTIR) spectra, and we used the ATR-transmittance measurement technique. The following parameters were used for the analysis: resolution, $4.0 \mathrm{~cm}^{-1}$; sample scan time, 16 scan; background scan time, 16 scan; spectral region FT-IR, $4000-650 \mathrm{~cm}^{-1}$. The data were normalized by the mean values, and in the infrared spectrum of each sample, three principal components were analyzed. Each measurement was performed in triplicate.

Five catheter samples were analyzed, with one sample from a new catheter and four from catheters that were reprocessed one, two, three, or seven times. Samples were cut into small pieces of about $5 \mathrm{~cm}$.

\section{Statistical analysis}

A descriptive statistical analysis of the variables of interest of the study was performed. In addition, graphics were built demonstrating the results.

\section{Results}

\section{Electron microscopy description}

No interruptions were seen in the edges of new catheter samples. In samples that went through a second reprocessing, the edges showed polymer wear and interruptions under $1,000 \times$ and 2,000 $\times$ magnifications. The samples that were reprocessed three times showed many orifices under $1,000 \times$ magnification and material wear and wrinkled areas at several points, which were best visualized under 2,000 and 4,000 $\times$ magnifications. The samples that were reprocessed seven times showed interruption clusters under 2,000 and 4,000 $\times$ magnifications. Figure 1 shows examples of catheters modifications.

\section{External side}

The analysis of the external side showed that the new catheter samples remained intact under 70,200, 500 and 1,000 $\times$ mag- 


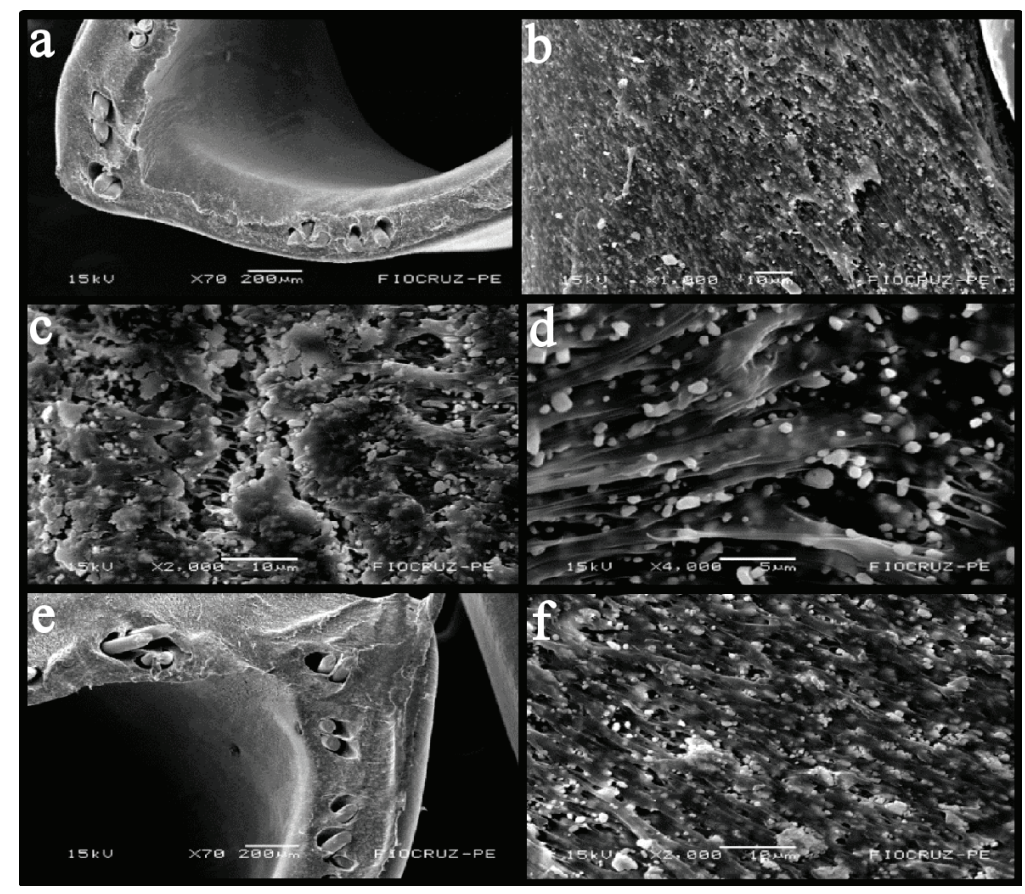

Figure 1. Scanning electron microscopy. (a) $70 \times$. (b) 1,000 ×. Edge: the second reprocessing (rougher surface, with porosity, stainless steel wires, peeling of the inner layers, micropores and small holes). (c) 2,000 ×. (d) 4,000 ×. Edge: the third reprocessing (disordered cracks, cracks, holes, grooves, microcracks and granular appearance). (e) $70 \times$. (f) 2,000 ×. Edge: the seventh reprocessing (roughness, porosity, stainless steel wires, holes, peeling and dots whitish, diffuse microcracks, granular appearance).

nifications and showed diffuse orifices under 4,000 $\times$ magnification. The sample that was reprocessed once showed 11 points of interruption in the polymeric layer, opacity of the polymeric material, and more than 10 dispersed particles under 500 and $1,000 \times$ magnifications. There was an increase of more than 30 orifices under $2,000 \times$ and more than 30 cracks under $4,000 \times$ magnification. The samples that were reprocessed twice presented two large scratches under $70 \times$ magnification, many hollows and cracks under $500 \times$ and $1,000 \times$ magnifications, 30 more cracks, and opacity of the polymeric material under $4,000 \times$ magnification. We observed increased fissures, the presence of whitish pigments, and several scratches in samples that were reprocessed three times. There were more than eight fissures, diffuse opacity of the material, 30 whitish pigments, and many scratches (an average of eight in each catheter) under $70 \times$ magnification. There were scratches, holes, cracks, and opacity under $200 \times, 500 \times, 1,000 \times, 2,000 \times$, and 4,000 $\times$ magnifications. The sample that was reprocessed seven times showed extensive longitudinal lines more than 10 times under $70 \times$, four darkened spots, 20 whitish spots, and general dryness. Dispersed whitish spots were observed under $200 \times$ magnification. More than 30 deep extensive cracks were seen under $500 \times, 1,000 \times, 2,000 \times$, and $4,000 \times$ magnifications. Figure 2 shows examples of catheters modifications.

\section{Internal side}

Analyses of the internal side of new catheter samples showed five interruptions under $70 \times$ magnification. The sample that was reprocessed once showed a dry area and 20 whitish spots. The samples that were reprocessed twice showed 10 fissures and three bulges. The samples that were reprocessed three times showed seven fissures and 10 interruptions. The sample that was reprocessed seven times showed five scratches, 30 diffuse spots, and seven interruptions. Disordered images and interlaced lines in the polymeric material were seen under $1,000 \times, 2,000 \times$, and 4,000 $\times$ magnifications. Figure 3 shows examples of catheters modifications.

\section{Infrared spectroscopy}

In the infrared spectrum shown in Figure 4, characteristic vibrations of the external side of angiographic catheters can be seen, as detailed in Table 1. The Figure 5 shows the spectra of new catheters and catheters that were reprocessed one, two, three, and seven times. No new peaks were found, but the intensity of the characteristic polyurethane peaks increased, which suggests a possible change in the chemical structures of the polymer.

\section{Carbonyl ratio}

The Figure 6 shows the carbonyl ratios of new catheters and catheters that were reprocessed one, two, three, and seven times. There is a directly proportional relationship between the 


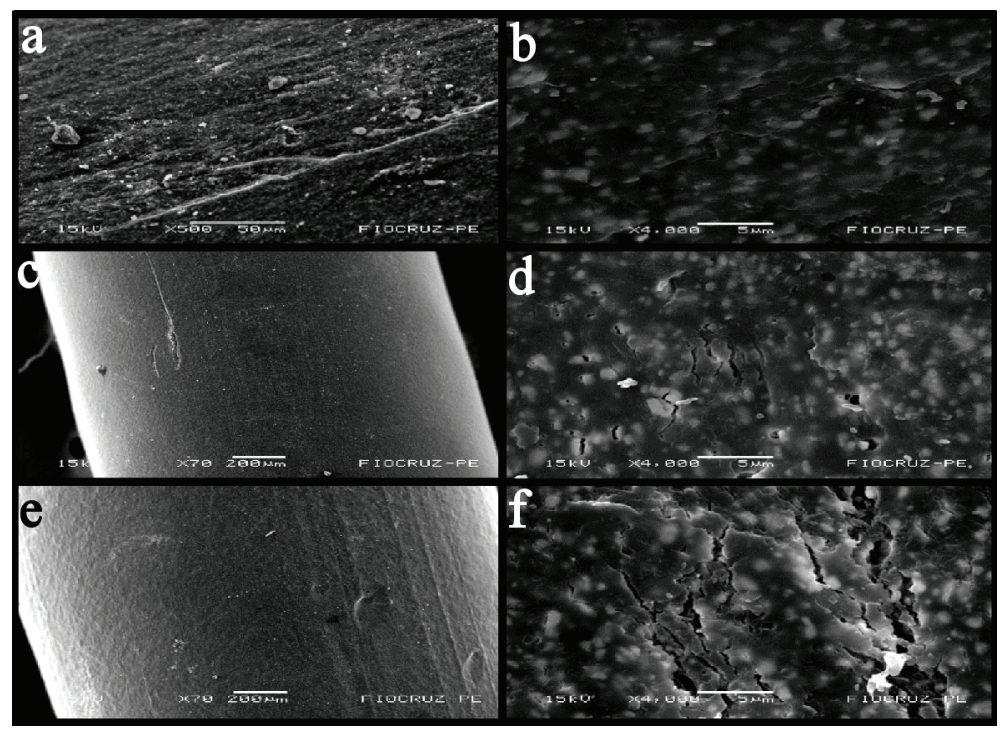

Figure 2. Scanning electron microscopy: (a) $500 \times$. (b) 4,000 $\times$. External side: the first reprocessing (desquamation, porosity, diffuse recesses, cracks, grooves and micro holes). (c) $70 \times$. (d) 4,000 $\times$. External side: the second reprocessing (fissures, porosities, scratches, cracks, micropores, and micro-holes, increased depressions, protrusions; diffuse dimples, disorder in the polymeric fibers). (e) $70 \times$. (f) 4,000 ×. External side: the seventh reprocessing (polymeric scaling, disordered slits, holes and grooves, recesses, protrusions, micro-holes, crevices, disordered depressions).

reprocessing number and the carbonyl ratio.

\section{Principal component analysis}

The Figure 7 shows the graph of the principal component analysis scores of new catheters and catheters that were reprocessed one, two, three, and seven times. The three principal components account for $98 \%$ of the spectral information. New and reprocessed samples were separated and reveal that a pos- sible change in catheter structure occurred after sterilization.

\section{Discussion}

The SEM is a type of electron microscope that is capable of producing high-resolution images of the surface of a sample, and examines the surface in such a way that the electron beam does not traverse the object. A narrow electronic beam is directed to the surface and scans the surface in a regular, side-to-

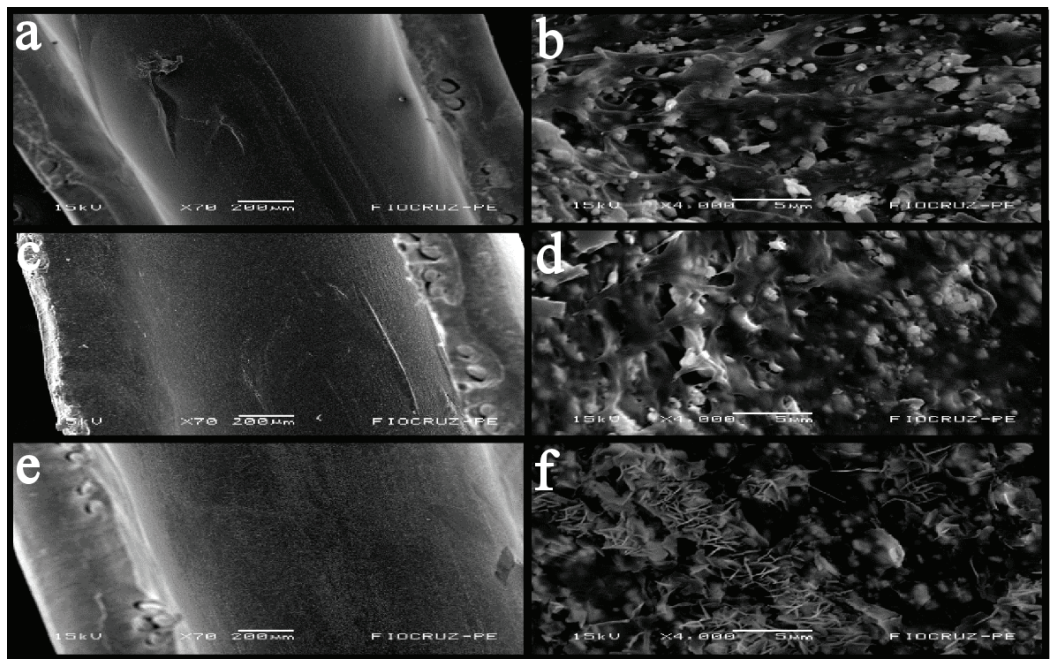

Figure 3. Scanning electron microscopy. (a) $70 \times$. (b) 4,000 ×. Internal side: new catheter (diffuse micropores, significant scratches, polymer desquamation, roughness and recess). (c) $70 \times$. (d) 4,000 ×. Internal side: the third reprocessing (microcracks, oxidative degradation, desquamation of the inner layers, disordered cracks, holes and whitish spots; deep and significant recesses). (e) $70 \times$. (f) 4,000 $\times$. Internal side: the seventh reprocessing (internal cracks, roughness, porosity, longitudinal microcracks, polymer desquamation, whitish spots, deep crevices, recesses and microcracks). 


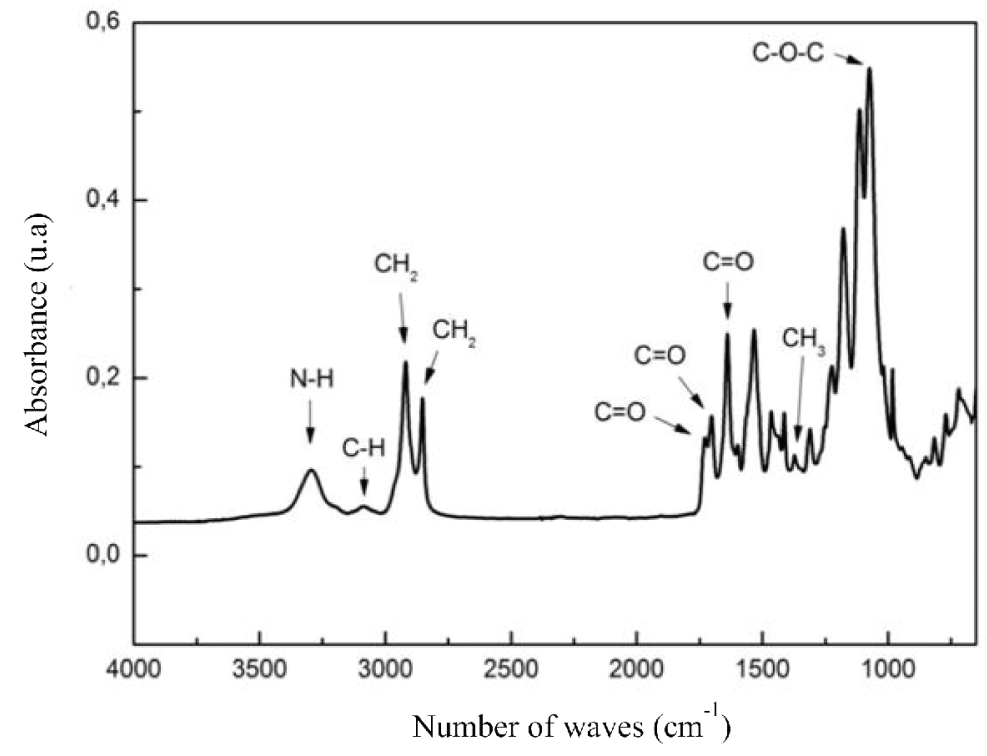

Figure 4. Angiographic catheter spectrum without reporcessing.

side pattern. When the scanning beam reaches the surface, it emits secondary electrons and synchronizes with the beam that produces the image on the monitor. Thus, a three-dimensional image of the sample surface can be constructed in the video, and micrographs can be obtained by photographing the image. The sample surface is then coated with a thin layer of metal, such as gold, white gold, carbon, to facilitate electron dispersion. A study that previously used SEM to analyze catheters showed that there was oxidative degradation of the polyamide polymer matrix when the material was exposed to hydrogen peroxide and the presence of micropores on the polymer surface, which became wider and led to the development of small micro-holes in the material. This analysis revealed increased wear of the angiographic catheters as the number of reprocessing increased [5].

Previous studies have also suggested that the presence of imperfections on the surface of catheters may be related to mechanical efforts and stresses that occur during the processing, handling, cleaning, and preparation of the material for sterilization. Lucas T.C. et al used scanning microscopy and found that the number of microcracks increased as reprocessing was repeated, which suggests that the material will have a shorter useful lifetime after each reprocessing. In addition, the single use of a catheter may result in the accumulation of biofilms and endotoxins within microcracks, which may lead to infections and the development of thrombi in patients. In our study, the SEM showed, at a structural level, that the reprocessed catheters were damaged, which corroborates some data in the literature. We identified that the catheters begin to change with the first reprocessing [4].

Infrared spectroscopy is one of the most important analytical techniques available today. One of the great advantages of this technique is that samples can be studied in almost any physical state. Liquids, solutions, pastes, powders, films, fibers, gases, and surfaces can be analyzed based on judicious choices and sampling preparation techniques. The introduction of FTIR spectrophotometers dramatically improved the quality of infrared spectra and minimized the time required to obtain data. FTIR is used to obtain absorption or emission infrared spectrum of solids, liquids, or gases. In this tech-

Table 1. Vibrations Characteristic of the Infrared Spectrum of the Angiographic Catherter

\begin{tabular}{lll} 
Number of waves $\left(\mathbf{c m}^{-\mathbf{1}}\right)$ & Absorption group & Types of deformation \\
\hline 3294 & $\mathrm{~N}-\mathrm{H}$ & Stretch $(\mathrm{N}-\mathrm{H})$ \\
3084 & $\mathrm{C}-\mathrm{H}$ aromatic & Stretch $(\mathrm{C}-\mathrm{H})$ \\
2920 & $\mathrm{C}-\mathrm{H}_{2}$ & Asymmetrical stretch $\left(\mathrm{CH}_{2}\right)$ \\
2853 & $\mathrm{CH}_{2}$ & Stretch $\left(\mathrm{CH}_{2}\right)$ \\
1734 & $\mathrm{C}=\mathrm{O}$ & Stretch $(\mathrm{C}=\mathrm{O})$ \\
1722 & $\mathrm{C}=\mathrm{O}($ free $\mathrm{CO})$ & Stretch $(\mathrm{C}=\mathrm{O})$ \\
1638 & $\mathrm{C}=\mathrm{O}($ bind $\mathrm{HCO})$ & Stretch $\mathrm{C}=\mathrm{O})$ \\
1363 & $\mathrm{CH}$ & Dobramento $\mathrm{CH}_{3}$ \\
1085 & $\mathrm{C}-\mathrm{O}-\mathrm{C}$ & Asymmetrical stretch $(\mathrm{C}-\mathrm{O}-\mathrm{C})$ \\
\hline
\end{tabular}




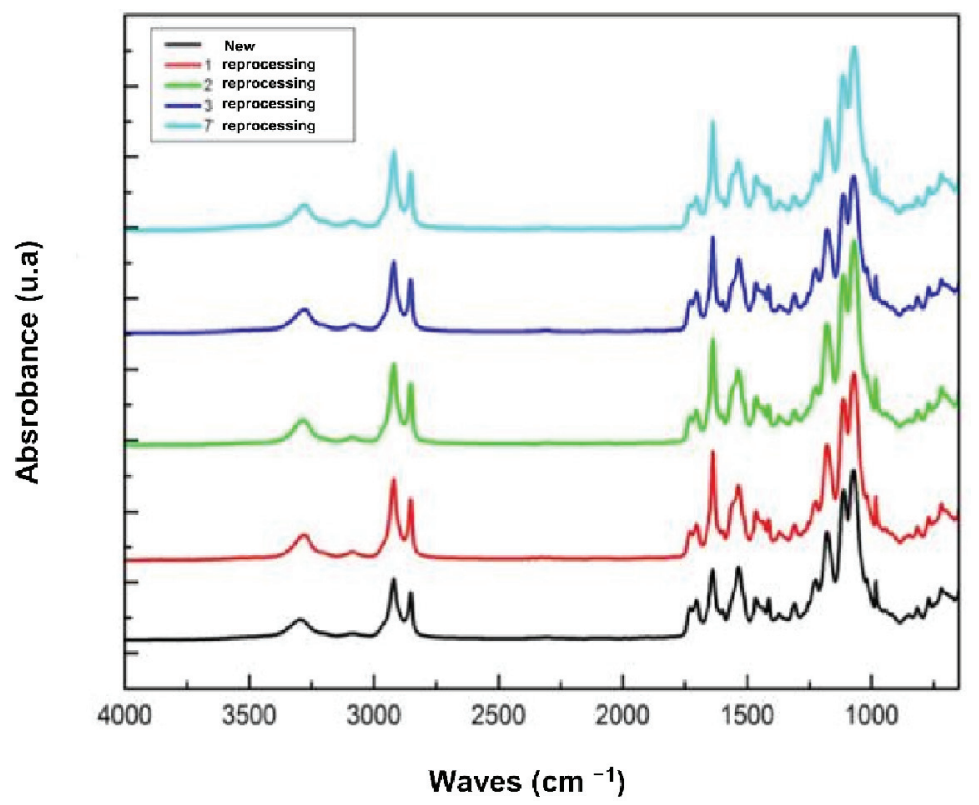

Figure 5. Infrared spectrum of angiographic catheters without and with the first, second, third and seventh reporcessing.

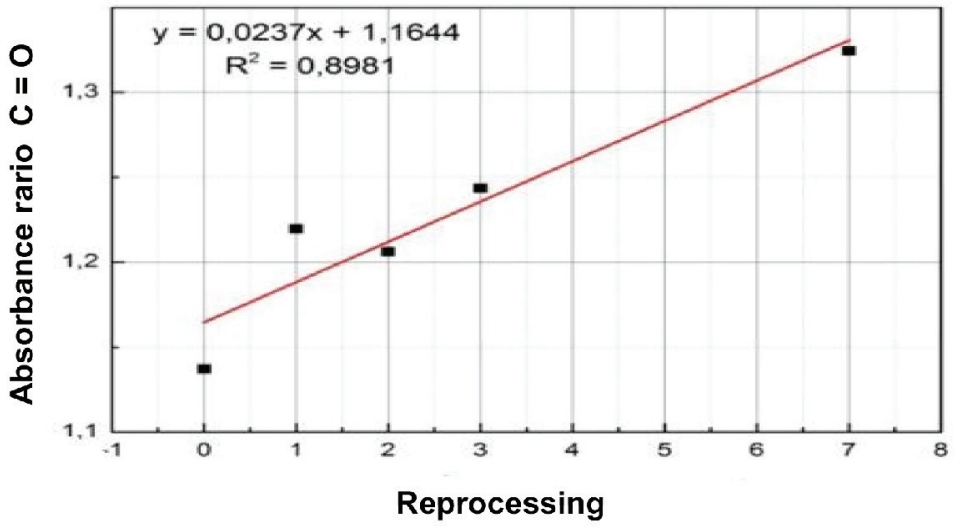

Figure 6. Absorbance ratio of carbonyl.

nique, a spectrometer simultaneously collects spectral highresolution data over a wide spectral range. FTIR is one of the most frequently recommended techniques for observing and evaluating degradation that occurs during catheter reprocess-

Scores

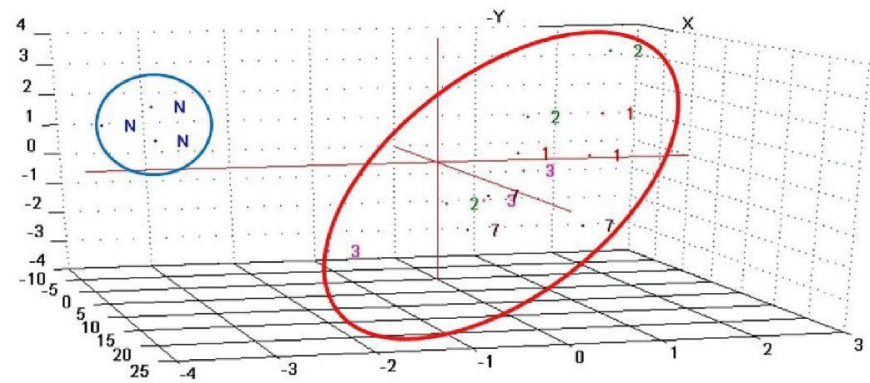

Figure 7. Component analysis scores of new catheters and catheters that were reprocessed one, two, three, and seven times. 
ing $[4,6]$.

The conventional infrared spectrum shows only the vibrations that lead to the rhythmic alteration of the dipole moment of a molecule. The alternating electric field, which is produced by the change of charge distributions that accompany the vibration, couples the molecular vibration with the oscillating magnetic field of the electromagnetic radiation, which results in radiant energy absorption. In the present study, the principal functional groups that compose the catheters, polyurethane and polyamide, were identified in the FTIR spectra. It is important to emphasize that since the spectroscopy method did not identify the internal surface of the catheter, polytetrafluoroethylene could not be analyzed. We observed that the reprocessing events were associated with changes in the components of the catheters $[7,8]$.

According to Oliveira D.C. et al, catheter materials can undergo surface oxidation, changes in hydrophilic properties, and drastic surface changes after sterilization with hydrogen peroxide. The increases in peak intensity that were found in our study in catheters that were reprocessed many times could be related to this phenomenon [9].

Oxygenated groups are considered a major factor in the loss of mechanical properties of materials, and a previous infrared study that evaluated polyurethane electrophysiology catheters that were reprocessed up to 10 times using Sterrad $\mathbb{R}$ $100 \mathrm{~S}$ concluded that the level of oxidation among the polymer chains was more evident and progressive in the spectra after five sterilizations. Infrared oxidation degradation studies of polyurethane blends showed that the absorption in the bridging carbonyl region increased significantly with the development of oxidative degradation, as evidenced by the prevalence of cross-links in relation to chain scission $[4,10,11]$.

The increased absorbance ratio in the bridging carbonyl region is mainly due to the cross-linking effect that localizes to the polar regions of molecules and increases the participation of carbonyls in the formation of hydrogen bonds. This effect increases hydrogen bonds in the polymer chain during scission, which promotes the organization and crystallization of molecules, due to greater mobility. Our study found that the higher are the number of reprocessing events, the higher are the carbonyl rates [4].

The combined SEM and infrared analyses reveal that reprocessing damages the integrity of coronary angiography catheters. Therefore, the reprocessing of such catheters is not recommended.

\section{Financial Support}

None.

\section{References}

1. Mansur Ade P, Favarato D. Mortality due to cardiovascular diseases in Brazil and in the metropolitan region of Sao Paulo: a 2011 update. Arq Bras Cardiol. 2012;99(2):755761.

2. Bueno MR, Ribeiro FG, Borges SR, Pavani LMD. Custo direto do reprocessamento de cateteres para estudos hemodinamicos. Estado de Sao Paulo, Rev Soc Cardiol. 2001;11:1-9.

3. Food and Drug Administration. FDA - Center for device and Radiological Health/CDRH. Coronary and peripheral arterial diagnostic catheters. 2006. http://www.fda.bov/ cdrh/od/guidance/1228.htm.

4. Lucas TC, Barbosa MP, de Oliveira AC. [Reprocessing validation of angiographic cardiac catheters: an evaluation of the functionality and integrity]. Rev Esc Enferm USP. 2010;44(4):947-955.

5. Tandon GP, Pochiraju KV, Schoeppner GA. Thermo-oxidative behavior of high-temperature PMR-15 resin and composites. J Med Devices. 2014;9:14-1209.

6. Stuart BH. Infrared Spectroscopy: Fundamentals and Applications. Analytical Techniques in the science (AnTs). Editora: Jonh Wiley \& Sons Ltd. 2004: p. 242.

7. Silverstein RM, Webster FX, Kiemle J. Identificacao Espectrometrica de Compostos Organicos: LTC - Livros Tecnicos e Cientificos Editora SA, 6a. Edicao, Rio de Janeiro. 2000.

8. Griffiths P, De Hasseth JA. Fourier Transform Infrared Spectrometry. Wiley-Blackwell. May, 2007.

9. Oliveira AC, Lucas TC. Validacao e limitacoes dos materiais de uso unico. On line Brazilian Journal of Nursing. 2008;7:1. http://www.uff.br/objnursing/index.php/nursing.

10. Callister WD. Materials science and engineering. Jonh Whiley \& Sons Ltd, 7a. Ed, New York, 2007.

11. Canevarolo J, Sebastiao V. Ciencia dos polimeros: um texto basico para tecnologos e engenheiros. Sao Paulo: Artliber Editora. 2004: p. 183. 